\title{
The antitumor and antimetastatic effects of N-trimethyl chitosan-encapsulated camptothecin on ovarian cancer with minimal side effects
}

\author{
LINA ZHOU ${ }^{1}$, LICHENG DU ${ }^{1,3}$, XIANCHENG CHEN $^{2}$, XINGYI LI $^{2}$, ZHIYONG LI $^{2}$, YUAN WEN ${ }^{2}$, \\ ZHENGYU LI ${ }^{1}$, XIANG HE ${ }^{1}$, YUQUAN WEI ${ }^{2}$, XIA ZHAO ${ }^{1}$ and ZHIYONG QIAN ${ }^{2}$ \\ ${ }^{1}$ Department of Gynecology and Obstetrics, West China Second Hospital, Sichuan University, Chengdu; \\ ${ }^{2}$ State Key Laboratory, Biotherapy and Cancer Center, West China Hospital, Sichuan University, Chengdu; \\ ${ }^{3}$ Shandong Provincial Hospital of Shandong University, No. 324 Jing Wu Road, Jinan, Shandong, P.R. China
}

Received March 29, 2010; Accepted May 5, 2010

DOI: $10.3892 /$ or_00000940

\begin{abstract}
Lymphatic metastasis plays a critical role in ovarian cancer, indicates poor prognoses and correlates to the majority of cancer deaths. Camptothecin derivatives exhibit promising activity for the treatment of solid tumors because of its specific inhibition of eukaryotic DNA topoisomerase I. Yet, its application is hindered due to extreme water insolubility and severe side effects. It is essential to establish an efficient and safe protocol for the administration of camptothecin versus tumor metastasis and growth. In the current research, we encapsulated camptothecin with $\mathrm{N}$-trimethyl chitosan (CPT-TMC) to increase its water-solubility and lower its side effects, and tested it on a high potential lymphogenous metastatic model of human ovarian cancer. In the prophase study, we successfully transfected SKOV3 cells with VEGF-D recombinant plasmid DNA (pcDNA3.1(+)/VEGF-D) to construct a cell line named SKOV3/VEGF-D and establish a feasible lymphogenous metastatic model. The antitumor and antimetastatic activities of CPT-TMC were evaluated in nude mice subcutaneously inoculated with SKOV3/VEGF-D cells at the left hindlimb claw pad. The tumor-bearing mice were divided randomly into four groups and treated twice per week for three weeks. Evan's Blue Dye was used to delineate
\end{abstract}

Correspondence to: Professor Xia Zhao, Department of Gynecology and Obstetrics, West China Second Hospital of Sichuan University, No. 20, Section 3, South People's Road, Chengdu 610041, Sichuan, P.R. China

E-mail: xia-zhao@126.com

Professor Zhiyong Qian, State Key Laboratory, Biotherapy and Cancer Center, West China Hospital, Sichuan University, Keyuan Road 4 No. 1, Gaopeng Street, High Technological Development Zone, Chengdu 610041, Sichuan, P.R. China

E-mail: anderson-qian@163.com

Abbreviations: CPT, camptothecin; NS, normal saline; LN, lymph node; LYVE-1, lymphatic vessel endothelial HA receptor-1; MMP-9, matrix metalloproteinase-9

Key words: lymphogenous metastasis, lymphangiogenesis, camptothecin, $\mathrm{N}$-trimethyl chitosan, ovarian cancer functional lymphatic vessels. Lymphatic metastasis rates were detected by hematoxylin and eosin (HE) staining. Expression of VEGF-D and MMP-9 were investigated by immunohistochemistry. In contrast to controls, administration of CPTTMC achieved effective inhibition in primary tumor volume and lymphogenous metastasis, yet without apparent systemic toxic effects. These effects were associated with simultaneously down-regulated VEGF-D and MMP-9 expression, significantly decreased tumor-associated lymphatic and blood sprouts, tremendously reduced systemic toxic effects, dramatically increased tumor apoptotic index. Our data indicate that CPT-TMC is superior to CPT by maximizing its anticancer and antimetastatic activities with minimal toxicity on hosts. CPT-TMC may become a potentially therapeutic strategy against human advanced ovarian cancer.

\section{Introduction}

Ovarian cancer accounts for the highest mortality rate among gynecological malignancies, making it the greatest threat to the health of women. Tumor cells spread to local lymph nodes, as an important indicator of tumor aggressiveness, is often an early event in metastatic disease (1). Metastasis via the lymphatic vessels plays a critical role for dissemination of tumor cells to sentinel lymph nodes in ovarian cancer, and indicates poor prognoses (2).

Although most primary tumors can be surgically removed, high incidence of lymphogenous metastasis and recurrence are still considerable aspects of ovarian cancer patients. Recent experimental evidence recognized that lymphangiogenic growth factor, especially the vascular endothelial growth factor-D (VEGF-D), which is thought to be involved in inducing tumor-associated lymphangiogenesis (3), could promote cancer cells spread to regional lymph nodes (4-7). In the prophase of the study, we successfully transfected the human ovarian serous cystadenocarcinoma SKOV3 cells with recombinant plasmid pcDNA3.1(+)/ VEGF-D (SKOV3/ VEGF-D) to establish a feasible high lymphogenous metastatic mouse model, and aimed to introduce it to experimental therapy research.

Even in advanced stages, ovarian cancer is still sensitive to a variety of chemotherapeutics. This highlights the need to 
develop reliable and efficacious chemotherapies that can inhibit metastasis and prolong survival time with minimal toxicity to patients.

Camptothecin, a pentacyclic cytotoxic alkaloid and a novel effective anticancer drug originally isolated from Camptotheca acuminata Decne in 1966 (8), is believed to act by stabilizing the topoisomerase I-induced DNA single strand break, so as to preventing religation (9). As a strong inhibitor of the DNA-replicating enzyme topoisomerase I (10), camptothecin has many novel activities, such as antitumor, antiproliferative effects and induces cell apoptosis in a large amount of cancer cell lines, especially in human xenografts of colon, lung, breast, ovarian, and melanoma cancers (11-14). Additionally, camptothecin also showed promising potential of antimetastasis in a broad spectrum of cancers, such as melanoma, ovarian cancer, colon, pancreatic cancer (15-18). These inspiring data suggested that camptothecin could be developed as a promising therapeutic impact on cancer progression and metastasis.

However, the extreme water insolubility and severe side effects hampered the application of camptothecin. In order to conquer the problems, our laboratory used N-trimethyl chitosan, a promising drug vector, to encapsulate camptothecin and named it as CPT-TMC. The present study was aimed to evaluate whether camptothecin could overcome tumor progression and lymphogenous metastasis in ovarian cancer while we addressed its disadvantages by encapsulating it in a suitable nanocarrier named N-trimethyl chitosan, which increased the drug solubility and minimized its systemic toxic effects.

In this study, CPT-TMC provided tremendous advantages in antitumor and anti-lymphatic metastasis in terms of significantly enhanced the inhibition in tumor growths and lymphatic metastases, down-regulated the expression of VEGF-D and reduced toxic side effects. These data demonstrate that CPT-TMC may be an efficient and safe protocol for human advanced ovarian cancer.

\section{Materials and methods}

Preparation of SKOV3/VEGF-D ovarian cancer cell line. The SKOV3 cells, obtained from the American Type Culture Collection (ATCC, Manassas, VA), were seeded in 6-well plates $\left(1 \times 10^{5} /\right.$ well $)$ and incubated at $37^{\circ} \mathrm{C}$ in $5 \% \mathrm{CO}_{2}$ overnight. Then cells were transfected with recombinant plasmid pcDNA3.1(+)/VEGF-D (obtained from the Division of Cancer Biotherapy in our laboratory) by lipofectamin 2000 reagent (Invitrogen). Transfected cells were then maintained in cell culture medium RPMI-1640 (Gibco) containing $10 \%$ fetal bovine serum and $400 \mu \mathrm{g} / \mathrm{ml} \mathrm{G} 418$ for three weeks, followed by G418 antibiotic resistance selection. After screening, the stably transfected cell clones (SKOV3/VEGF-D) were obtained.

$R T$-PCR. RT-PCR was employed to verify the stable expression of VEGF-D in tumor cells. Total RNA was extracted from $1 \times 10^{7}$ tumor cells (SKOV3 cells, SKOV3 cells transfected with empty plasmid, SKOV3 cells transfected with recombinant plasmid pcDNA3.1(+)/VEGF-D, which named as SKOV3, SKOV3/pcDNA, and SKOV3/ VEGF-D, respectively), performed as the introduction of
TRIzol (Invitrogen) and detected by spectrophotometric analysis. The following specific primer pairs were used: 5'-GC AAG CTT ATG TAT GGA GGA TGG GGA ATG-3' (VEGFDFOR). 5'-CG TCT AGA TCA AGG GTT CTC CTG GCT G-3' (VEGFDREV) (amplifies nucleotides 283-1359 of mouse VEGF-D, GenBank accession number NM-010216 GI: 6753873).

Tumor models. The research protocol was reviewed and approved by the Institutional Animal Care and Treatment Committee of Sichuan University. Female athymic BALB/c nude mice, 6-8-week old, were maintained in pathogen-free conditions and fed sterile chow. Before inoculation, mice were randomly assigned into three groups (six per group). Three kinds of cells (SKOV3, SKOV3/pcDNA, SKOV3/VEGF-D) were inoculated subcutaneously into the hind left footpad of recipients $\left(2 \times 10^{6}\right.$ cells resuspended in $50 \mu 1$ of PBS), respectively. Tumor growth and mice behavior or physical appearances were evaluated consecutively for two months.

Preparation of CPT-TMC. CPT-TMC was prepared by combination of microprecipitation and sonication. Briefly, $6 \mathrm{mg} / \mathrm{ml}$ of camptothecin was first prepared by dissolving $30 \mathrm{mg}$ camptothecin into $5 \mathrm{ml}$ dimethyl sulfoxide (DMSO) solution. Then TMC was dissolved in water solution at the concentration of $5 \mathrm{mg} / \mathrm{ml}$. Subsequently, $0.1 \mathrm{ml}$ of camptothecin solution was added dropwisely into $2 \mathrm{ml}$ of TMC solution at $4^{\circ} \mathrm{C}$. The obtained colloid solution was ultrasonicated for 10 min keeping the temperature at $4^{\circ} \mathrm{C}$. Finally, the colloid solution was dialyzed against water using a membrane with a molecular weight cut-off of 8000-14000 (Solarbio, China) for 3 days, and then the solution was centrifuged at $10000 \mathrm{x} \mathrm{g}$ for $10 \mathrm{~min}$ to remove insoluble camptothecin.

Cytotoxicity assay in vitro. The cytotoxicity of CPT-TMC on SKOV3/VEGF-D cells was determined by MTT assay; and the absorbance at $490 \mathrm{~nm}$ was measured by using a multiwell plate reader (BioTek Instruments, Winooski, VT, USA). Effects of CPT-TMC, CPT and TMC on SKOV3/VEGF-D cell viability were assessed as percent cell viability in terms of media-only treated control cells (\% of control).

Treatment for high lymphogenous metastasis human ovarian cancer model. SKOV3/VEGF-D cells $\left(2 \times 10^{6}\right.$ cells resuspended in $50 \mu 1$ of PBS) were inoculated subcutaneously into the hind left footpad of recipients, which would later receive the following treatments. Before treatment, the tumorbearing mice were randomly divided into 4 groups $(5$ per group) when tumors were palpable: i) CPT-TMC (2.5 mg/kg); ii) CPT (2.5 mg/kg); iii) TMC (25 mg/kg); and iv) $0.9 \%$ NS. Treatment started 7 days after tumor cell challenged and administration via i.v. performed twice per week for 3 weeks. Tumor growth was monitored every 4 days and tumor volume was calculated as $0.52 \mathrm{x}$ length $\mathrm{x}$ width ${ }^{2}$.

Lymphangiography and lymphogenous metastasis. When tumors grew to $>1 \mathrm{~cm}$ in diameter, Evan's blue dye $(5 \mathrm{mg} / \mathrm{ml}$ in PBS) was infused intradermally into the tumor area after anesthesia to visualize the functional lymphatic vessels inside 
A

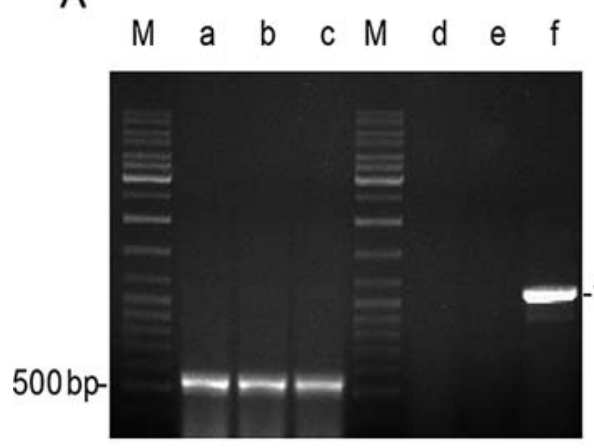

B

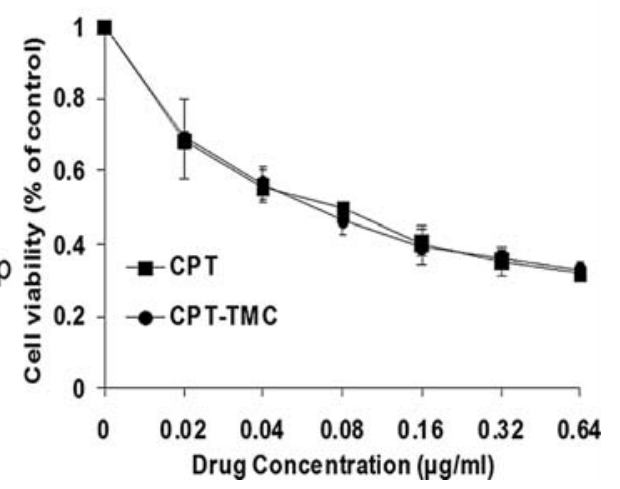

Figure 1. PT-PCR and MTT assay. (A) SKOV3 (lane a), SKOV3/pcDNA (lane b) and SKOV3/VEGF-D cells (lane c) showed the same positive band at about 500 bp (ß-actin); SKOV3 (lane d) and SKOV3/pcDNA cells (lane e) showed negative band at about 1000 bp (VEGF-D), but SKOV3/VEGF-D cells (lane f) showed strong positive band. (B) MTT showed both CPT-TMC and CPT significantly inhibited the viability of SKOV3/VEGF-D cells compared with TMC and media-only treated groups $(\mathrm{P}<0.05)$. No significant difference was observed between CPT-TMC and CPT group $(\mathrm{P}>0.05)$. Data are expressed as percentages of viable cells relative to media-only treated control cells at each drug concentration. Means $\pm S E(n=3)$, M, Marker.

and around the tumor masses (19). The lymphatic vessels specifically take up Evan's blue dye, whereas the blood vessels do not $(20,21)$. Multistep metastasis to LNs were evaluated with multilevel serial sections by hematoxylin and eosin (HE) staining and examined under microscope 8 weeks post tumor inoculation. The metastasis rates of LNs were assessed as the number of LNs with metastases out of the total number of LNs.

Immunohistochemistry staining. Sections of 3-5 $\mu \mathrm{m}$ thick were first deparaffinized and rehydrated. Then antigen retrieval was carried out by heating at $120^{\circ} \mathrm{C}$ in $10 \mathrm{mM}$ citrate buffer solution ( $\mathrm{pH}$ 6.0). Endogenous peroxidase activity was blocked by $3 \% \mathrm{H}_{2} \mathrm{O}_{2}$ and non-specific binding of reagents was quenched by $10 \%$ normal goat or rabbit serum. Then sections were incubated with primary antibodies, followed by incubating with biotinylated rabbit anti-goat/rat $\operatorname{IgG}$ and then streptavidin-biotin-horseradish peroxidase complex successively.

Quantification of lymphangiogenic and angiogenic microvessel density. To determine the lymphatic microvessel density (LMVD) and angiogenic microvessel density (MVD), sections were first scanned under a light microscope with low power magnification to identify the areas with the highest number of lymphatic or angiogenic vessels. Vessels within five highpower fields of these areas, with a clearly defined lumen or well defined linear vessel shape, were taken into account for lymphatic or blood microvessel and an average count was recorded.

Apoptotic analysis. Terminal deoxynucleotidyl transferasemediated dUTP nick-end labeling (TUNEL) was performed to analyse the inducible effect on apoptosis by CPT-TMC according to the manufacturer's protocol (Promega). Pyknotic nuclei stained with dark green, as viewed by fluorescence microcopy, were thought to be TUNEL-positive nuclei. Percent apoptosis was determined by counting the number of TUNEL-positive cells and dividing by the total number of cells in the field (five high-power fields/slide).
Toxicology analysis. Relevant indexes such as weight loss, anorexia, diarrhea, skin ulceration, ruffled fur or toxic death were observed consecutively for $>2$ months to evaluate the potential side effects and toxicity of CPT-TMC. Sections of various organs (heart, liver, spleen, lung, and kidney) were detected by HE staining under microscope.

Statistical analysis. Numerical values are described as means \pm one standard error (SE). One-way analysis of variance (ANOVA) was used to determine statistical significance in comparisons of experimental data among different groups. Differences were considered significant at $\mathrm{P}<0.05$.

\section{Results}

Stable expression of VEGF-D in SKOV3/VEGF-D cell lines. Expression of VEGF-D in SKOV3, SKOV3/pcDNA, and SKOV3/VEGF-D cells was expanded and analysed by RTPCR, respectively. Strong positive expression of VEGF-D was detected in SKOV3/VEGF-D cells versus the two controls, and verified by DNA sequencing (Fig. 1A).

VEGF-D promoted tumor growth and lymphangiogenesis. Significant differences in the size of primary tumor were observed in SKOV3/VEGF-D group versus control groups $(\mathrm{P}<0.05)$ from day 25. LMVD was evaluated by LYVE-1 staining. SKOV3/VEGF-D group displayed significant increase in LMVD (104.92 \pm 17.95$)$, compared with SKOV3/ pcDNA (33.71 10.29$)$ and SKOV3 (27.64 \pm 8.37$)$ groups. SKOV3/VEGF-D group showed much more metastasis tendency in sentinel LNs compared with the two controls

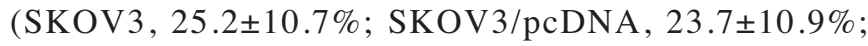
SKOV3/VEGF-D, 86.1 $13.7 \%$ ) two months after tumor cell challenge.

Anti-proliferation by CPT-TMC in vitro. MTT assay revealed that $\mathrm{CPT}-\mathrm{TMC}$ and $\mathrm{CPT}$ resulted in significant reduction in cell viability of SKOV3/VEGF-D cells compared with TMCtreated and media-only treated cells $(\mathrm{P}<0.05)$. No significant difference was observed between CPT-TMC and CPT, or 


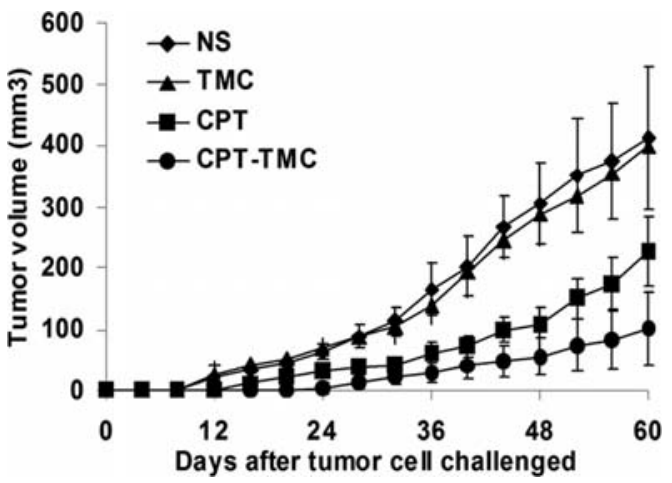

Figure 2. Tumor suppression. CPT-TMC showed significant differences in tumor volume compared with NS, TMC and CPT groups $(\mathrm{P}<0.05)$ There was no statistical difference between TMC and NS groups $(\mathrm{P}>0.05)$. Data shown as means $\pm \mathrm{SE}$. between TMC-treated and media-only treated cells $(\mathrm{P}>0.05$ Fig. 1B).

CPT-TMC inhibited tumor growth in vivo. Treatment with CPT-TMC significantly inhibited the primary tumor growth in comparison with controls $(\mathrm{P}<0.05$, Fig. 2$)$. Complete tumor regression occurred in one of the five mice administrated with CPT-TMC. CPT-TMC resulted in $>70 \%$ inhibition in tumor volume compared with NS and TMC groups two months after tumor inoculation. No significant difference was observed between TMC and NS groups ( $\mathrm{P}>0.05)$. Statistical significance was observed between CPT-TMC and CPT groups in the ultimate stage of this study.

Lymphangiography and reduction of lymphogenous metastasis. The intravital peritumoral lymphangiogram
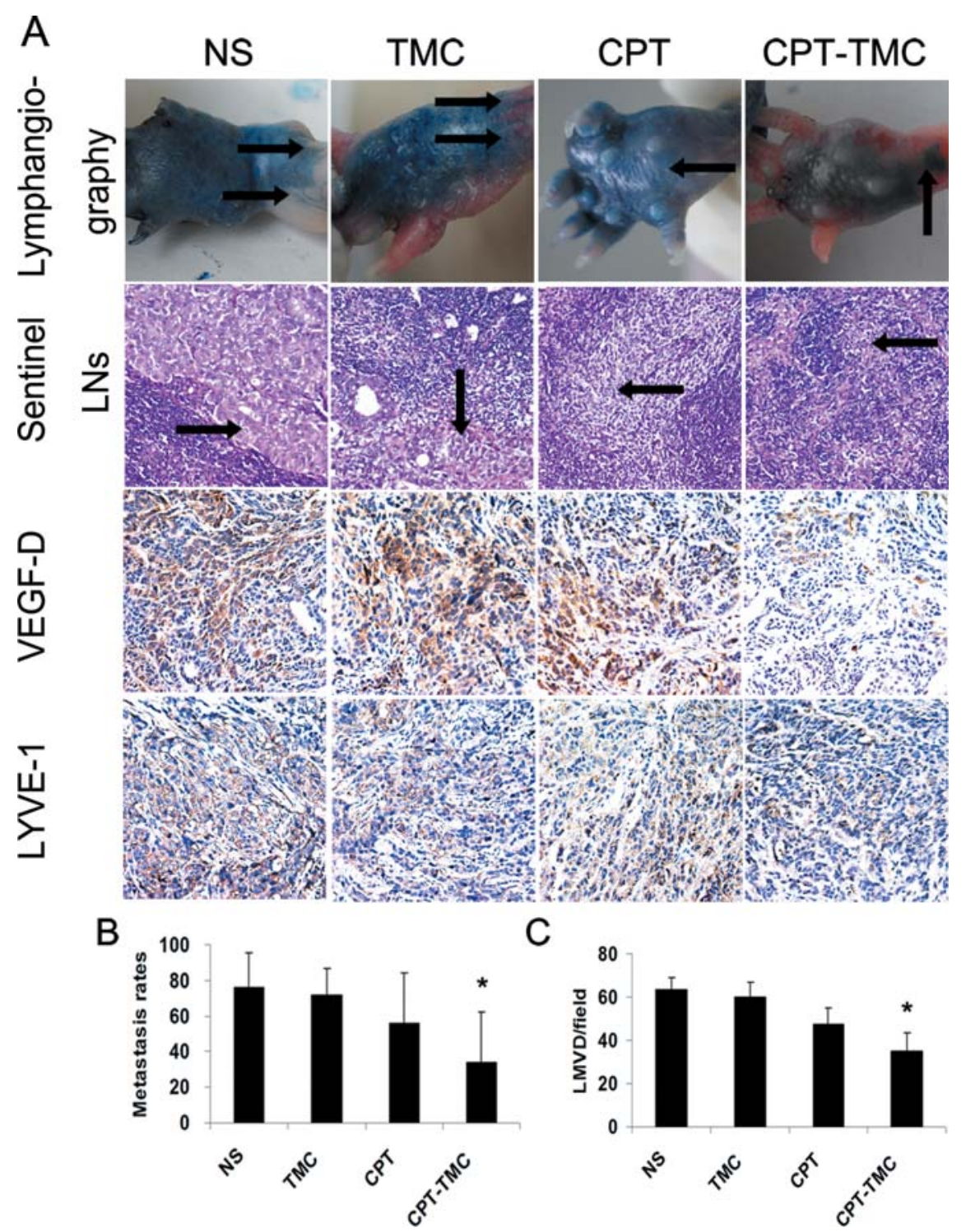

Figure 3. (A) Top panel, Evan's Blue dye was injected to delineate lymphatic vessels. Plentiful peritumoral lymphatic vessels emerged around tumors in NS, TMC and CPT (arrow, lymph flow), yet only a few in CPT-TMC group. Upper-middle panel, microscopic images showed metastasis of sentinel lymph node of NS, TMC, CPT and CPT-TMC groups. The metastasis foci are indicated by arrows. Lower-middle pane, VEGF-D expression in primary tumor showed much stronger staining in control groups versus a majority of CPT-TMC-treated tumor cells as well as interstitium. Bottom panel, LYVE-1 staining revealed that numerous newborn lymphatic microvessels among tumor cells of control groups, yet only sparse in CPT-TMC group. (B) Metastasis rates in sentinel lymph node showed CPT-TMC group had a much lower metastasis tendency than control groups ( ${ }^{*} \mathrm{P}<0.05$ versus NS and TMC groups). (C) CPT-TMC displayed significant inhibition of LMVD ( ${ }^{*} \mathrm{P}<0.05$ versus the three controls). Results expressed as the means $\pm \mathrm{SE}$. 


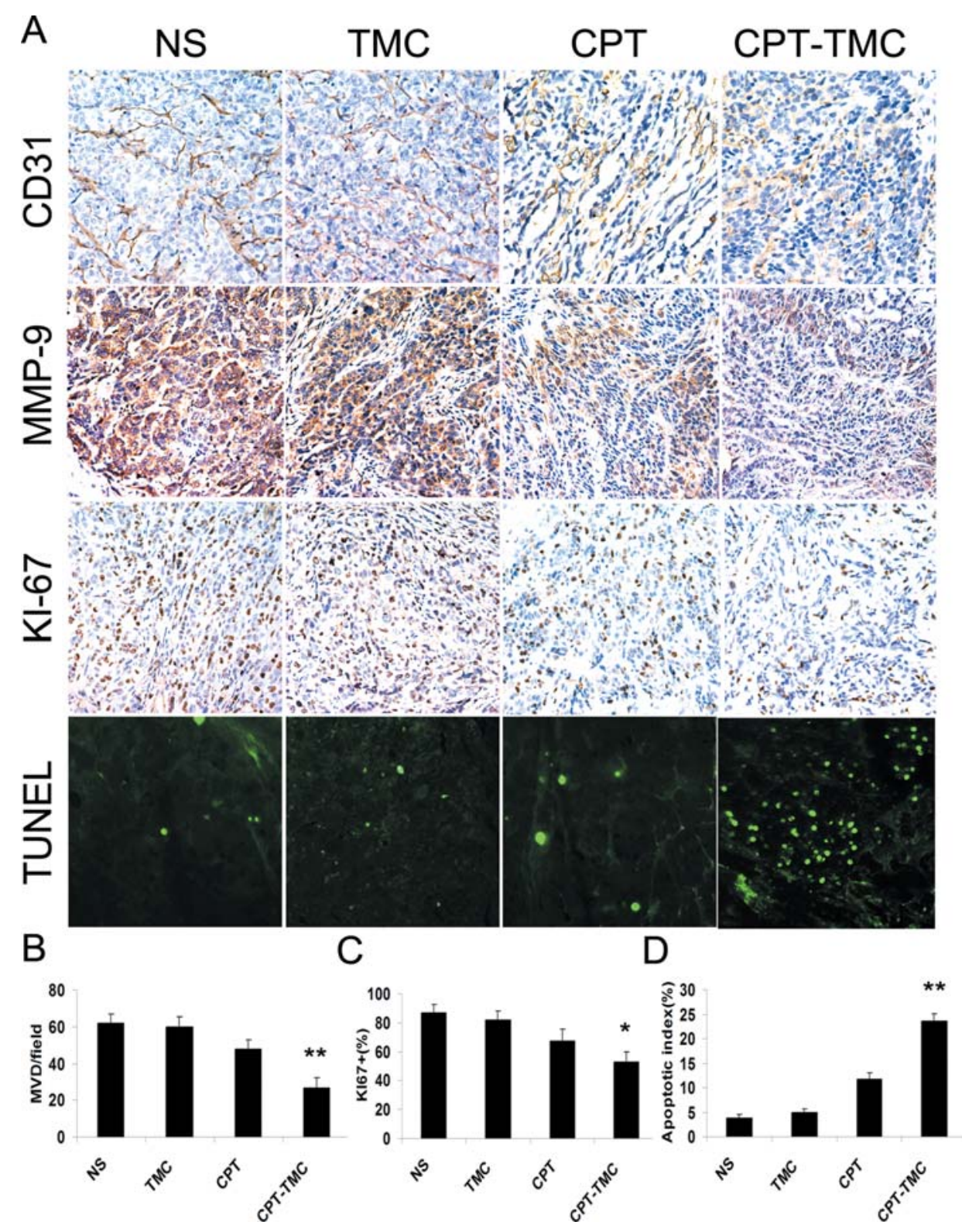

Figure 4. (A) Top panel, CD31 staining revealed plentiful nascent microvessels in control groups, but few in CPT-TMC group. Upper-middle panel, MMP-9positive cells were rich in NS and TMC groups, yet not in CPT-TMC group. CPT reduced the expression to some extent. Lower-middle panel, many KI-67positive cells were identified in NS and TMC groups compared to CPT and CPT-TMC groups. Bottom panel, TUNEL assay showed only a few positive nuclei in tumor tissues of controls, but plentiful in CPT-TMC group. (B) CPT-TMC significantly reduced CD31-positive microvessels compared with the controls. (C) Percentages of KI-67-positive nuclei showed tremendous reduction occurred in CPT-TMC group. (D) CPT-TMC markedly increased the percent apoptosis versus controls. Data are presented as means $\pm \mathrm{SE} .\left({ }^{*} \mathrm{P}<0.05 ;{ }^{* *} \mathrm{P}<0.01\right)$.

exhibited abundant dilated lymphatic vessels with sprout clumps observed in NS, TMC and CPT groups, yet seldom in CPT-TMC group (Fig. 3A). Consecutive histopathological analysis of LNs showed that metastasis were found in all group two months after the tumor cell challenge, but CPTTMC significantly suppressed the progression of metastases into sentinel lymph nodes versus NS and TMC controls $(\mathrm{P}<0.05$, Fig. 3A and $\mathrm{B})$. However, no statistical significance was found between CPT-TMC and CPT groups.

CPT-TMC inhibited VEGF-D expression and lymphangiogenesis. Immunohistochemistry showed that distinct clusters of VEGF-D-positive cells presented in the tumor tissues of different controls, which exhibited extensive invasion and metastases to remote multistep LNs and organs, yet only a few could be identified in CPT-TMC group (Fig. 3A). LYVE-1 staining exhibited that plentiful nascent lymphatic vessels were identified in NS and TMC groups, yet seldom in CPT-TMC group (Fig. 3A). CPT suppressed the expression of VEGF-D and LYVE-1 to some extent. The most significant reduction in VEGF-D expression and LMVD occurred in the CPT-TMC group compared with the other three groups $(\mathrm{P}<0.05$, Fig. 3C). No significant difference was found between TMC and NS groups ( $\mathrm{P}>0.05)$.

CPT-TMC suppressed angiogenesis and down-regulated MMP-9 expression. MVD was examined by CD31 staining. Numerous newborn blood capillaries could be observed in 
control groups, yet only a few CD31-positive staining appeared in CPT-TMC group (Fig. 4A and B). Matrix metalloproteinase-9 (MMP-9)-positive cells which were originally implicated in cancer progression, invasion and metastasis, were identified in the tumor tissues of different controls, yet only a few in CPT-TMC group (Fig. 4A).

CPT-TMC inhibited proliferation and increased apoptosis in vivo. KI-67 staining indicated that dramatically reduction occurred in CPT-TMC-treated group compared with controls $(\mathrm{P}<0.05$, Fig. 4A and $\mathrm{C})$. The number of KI-67-positive cancer cell nuclei was counted as a ratio of immunoreactive positive cells to the total number of cells counted. TUNEL assay revealed many strongly TUNEL-positive nuclei in CPT-TMCtreated tumor tissues, yet rarely in control groups (Fig. 4A). Apoptotic indexes showed only CPT-TMC therapy resulted in a significant increment of apoptotic index versus controls $(\mathrm{P}<0.01$, Fig. 4D).

Toxicity observation. Weight of mice was monitored every 4 days and considered as a parameter for evaluation of physical status, anorexia, or cachexia, and there was no evidence of toxicity in terms of body weight loss in CPT-TMC group. Furthermore, HE staining of the heart, liver, spleen, lung, kidney, intestine and bone marrow did not reveal any significant difference among CPT-TMC-treated group and control groups.

\section{Discussion}

Ovarian cancer causes the most cancer-related death in gynecological malignancies (22), owing to its high incidence of lymphogenous metastasis and recurrence, as well as the severe toxic side effects of available chemotherapeutic agents. Besides the well informed blood vessels, lymphatic vascular system provides another suitable pathway for the spread of cancer cells (2). Multiple lines of evidence have demonstrated that cancer cells spread to local lymph nodes, as a novel prognostic parameter for the metastatic risk, occurring in the early stage of the progression in many types of human neoplasms (23).

Although lymphatic vessels are important for tumor dissemination, little is known on the major molecular mechanisms of how lymphangiogenesis facilitates metastasis. Recent research indicates that VEGF-D, as one of the strongest agents promoting lymphangiogenesis and metastasis spreading of tumor cells to lymph node (24), is thought to be an important prognostic factor predicting short survival $(25,26)$. Many studies showed that cancer cells may exploit the vascular systems by spontaneously expressing protein growth factors, such as VEGF-C and VEGF-D, the best characterized vascular growth factors which can alter the normal pattern of angiogenesis, increase vascular leakage and drive lymphangiogenesis thus creating conduits for facilitating tumor metastasis (27). In the prophase of the current study, we successfully transfected SKOV3 cells with recombinant plasmid pcDNA3.1(+)/VEGF-D and injected them subcutaneously into the hind left footpad of recipients to establish a high lymphogenous metastasis mouse model of human ovarian cancer. We observed that
VEGF-D could dramatically promote tumor growth, induce lymphangiogenesis, and increase lymphatic metastasis. Because of these encouraging data, we used this promising high lymphatic metastatic mouse model of human ovarian cancer in our research.

Lymphogenous metastasis, recurrence and severe side effects of chemotherapy lead to low 5-year survival (28) and became the major obstacle to clinical treatment in ovarian cancer. However, even in advanced stages, ovarian cancer is still sensitive to a variety of chemotherapeutics. This highlights the need to develop reliable, low toxicity and efficacious chemotherapies againts lymphatic metastasis to prolong survival.

We selected camptothecin, a specific inhibitor of the DNA-replicating enzyme topoisomerase I (10), as the model drug for this study. It has multiple biological activities as described before. However, the extreme water insolubility and unpredictable side effects, as the major drawbacks of camptothecin, hindered its application in cancer therapy. The pharmacologically important lactone ring of camptothecin and its analogs are apt to bind albumin when in the presence of human serum albumin, resulting in conversion of the active drug to the inactive carboxylate form (29). These disadvantages impose severe pharmacokinetic limitation on the systemic use of camptothecin and related compounds. To solve these problems, an advisable drug delivery system is urgently needed to optimize its beneficial therapeutic efficacy and limit the disadvantages.

Chitosan, known as a natural aminopolysaccharide obtained by hydrolysis of chitin, has attracted much attention as a potential pharmaceutical excipient for drug delivery. It has favorable biological properties, such as safe, effective, biocompatibility, biodegradability, homogeneity, positive charge, non-toxicity, and bioadhesivity $(30,31)$. However, this polymer is only soluble in acidic environments with $\mathrm{pH}$ value lower than 6.0. Therefore, we chose a water-soluble quaternized cationic chitosan derivative, $\mathrm{N}$-trimethyl chitosan chloride (TMC), which maintained all the advantages of chitosan and can be soluble in the entire $\mathrm{pH}$ range. Many studies proved that TMC could facilitate the paracellular transport of drugs by effectively opening the intercellular tight junctions (32). These attributes have so far been beyond the reach of any other type of cell vehicles. This non-absorbable and non-toxic polymer represents an attractive technology platform for favoring the uptake and retention of camptothecin in cancer. We used this feasible drug carrier to encapsulate camptothecin and named it as CPT-TMC, aimed to pave the way to improve the security of practical application of camptothecin.

In the current study, we tested CPT-TMC on a high potential lymphatic metastasis model of ovarian cancer. CPT-TMC showed tremendous inhibition of advanced tumor growth and metastasis to multistep LNs yet without systemic toxic effects, by contrast, CPT, TMC and NS groups lack these attributes. Complete tumor regression occurred in one of the five mice administered with CPT-TMC. Emerging evidence shows that MMP-9 was associated with cancer invasion and progression $(33,34)$. In this study, quantitative histomorphometric analysis showed both VEGF-D-positive and MMP-9-positive expression in tumor tissues were 
remarkably down-regulated by CPT-TMC. Immunohistochemistry staining of tumor tissues with LYVE-1, CD31 and KI-67 revealed that LMVD, MVD and KI-67 positive cells were significantly reduced in CPT-TMC-treated group in contrast to other groups $(\mathrm{P}<0.05)$. These delectable results indicated that overexpression of VEGF-D increased the lymphatic sprouts and the potential of lymphogenous metastasis, yet down-regulated its expression could remarkably inhibit lymphangiogenesis and tumor growth. TUNEL assay showed significant increment of apoptotic index by CPT-TMC $(\mathrm{P}<0.05)$. With the drug administered in this dose and schedule, the histopathology of animal tissues revealed no obvious systemic toxicity. These results strongly suggest that CPT-TMC may successfully overcome lymphogenous metastasis in ovarian cancer.

MTT assay was carried out to confirm the toxicity effect of CPT-TMC on SKOV3/VEGF-D cells in vitro. The results showed that both CPT-TMC and CPT could significantly induce growth inhibition in SKOV3/VEGF-D cells, since the TMC itself (without camptothecin) produced no cytotoxicity according to the MTT assay. Our findings were in line with the anti-proliferative ability of camptothecin reported before (12).

In conclusion, our investigation suggested that CPT-TMC had displayed not only local anti-tumor ability but also strongly anti-lymphangiogenesis and anti-angiogenesis effects with low toxicity on the high lymphogenous metastatic model of ovarian cancer. CPT-TMC should be further investigated to pave the way for a new powerful therapeutic weapon against advanced human ovarian malignancies, particularly for patients with lymphogenous metastasis.

\section{Acknowledgements}

We thank Zhixing Cao for providing technical help. This study was supported by National Key Basic Research Program of China (2010CB529900).

\section{References}

1. Achen MG, McColl BK and Stacker SA: Focus on lymphangiogenesis in tumor metastasis. Cancer Cell 7: 121-127, 2005.

2. Van Trappen PO and Pepper MS: Lymphangiogenesis and lymph node microdissemination. Gynecol Oncol 82: 1-3, 2001 .

3. Stacker SA, Caesar C, Baldwin ME, Thornton GE, Williams RA, Prevo R, Jackson DG, Nishikawa S, Kubo H and Achen MG: VEGF-D promotes the metastatic spread of tumor cells via the lymphatics. Nat Med 7: 186-191, 2001.

4. Stacker SA, Baldwin ME and Achen MG: The role of tumor lymphangiogenesis in metastatic spread. FASEB J 16: 922-934, 2002.

5. He Y, Karpanen T and Alitalo K: Role of lymphangiogenic factors in tumor metastasis. Biochim Biophys Acta 1654: 3-12, 2004.

6. Stacker SA, Achen MG, Jussila L, Baldwin ME and Alitalo K: Metastasis: lymphangiogenesis and cancer metastasis. Nat Rev Cancer 2: 573-583, 2002.

7. Oliver $G$ and Detmar M: The rediscovery of the lymphatic system: old and new insights into the development and biological function of the lymphatic vasculature. Genes Dev 16: 773-783, 2002.

8. Gallo RC, Whang-Peng J and Adamson RH: Studies on the antitumor activity mechanism of action on cell cycle effects of camptothecin. J Natl Cancer Inst 46: 789-791, 1971.
9. Hsiang YH, Hertzberg R, Hecht S and Liu LF: Camptothecin induces protein-linked DNA breaks via mammalian DNA topoisomerase I. J Biol Chem 260: 14873-14878, 1985.

10. Herben VM, ten Bokkel Huinink WW and Beijnen JH: Clinical pharmacokinetics of topotecan. Clin Pharmacokinet 31: 85-102, 1996.

11. Van Hattum AH, Pinedo HM, Schluper HM, Erkelens CA, Tohgo A and Boven E: The activity profile of the hexacyclic camptothecin derivative DX-8951f in experimental human colon cancer and ovarian cancer. Biochem Pharmacol 64: 1267-1277, 2002.

12. Wang LM, Li QY, Zu YG, Fu YJ, Chen LY, Lv HY, Yao LP and Jiang SG: Anti-proliferative and pro-apoptotic effect of CPT13, a novel camptothecin analog, on human colon cancer HCT8 cell line. Chem Biol Interact 176: 165-172, 2008.

13. Knight V, Koshkina NV, Waldrep JC, Giovanella BC and Gilbert BE: Anticancer effect of 9-nitrocamptothecin liposome aerosol on human cancer xenografts in nude mice. Cancer Chemother Pharmacol 44: 177-186, 1999.

14. Lee DH, Kim SW, Suh C, Lee JS, Lee JH, Lee SJ, Ryoo BY, Park K, Kim JS, Heo DS and Kim NK: Belotecan, new camptothecin analogue, is active in patients with small-cell lung cancer: results of a multicenter early phase II study. Ann Oncol 19: 123-127, 2008.

15. Potmesil M, Vardeman D, Kozielski AJ, Mendoza J, Stehlin JS and Giovanella BC: Growth inhibition of human cancer metastases by camptothecins in newly developed xenograft models. Cancer Res 55: 5637-5641, 1995.

16. Matsuzaki T, Yokokura T, Mutai M and Tsuruo T: Inhibition of spontaneous and experimental metastasis by a new derivative of camptothecin, CPT-11, in mice. Cancer Chemother Pharmacol 21: 308-312, 1988 .

17. De Cesare M, Pratesi G, Veneroni S, Bergottini R and Zunino F: Efficacy of the novel camptothecin gimatecan against orthotopic and metastatic human tumor xenograft models. Clin Cancer Res 10: 7357-7364, 2004.

18. Sun FX, Tohgo A, Bouvet M, Yagi S, Nassirpour R, Moossa AR and Hoffman RM: Efficacy of camptothecin analog DX-8951f (Exatecan Mesylate) on human pancreatic cancer in an orthotopic metastatic model. Cancer Res 63: 80-85, 2003.

19. Chen XC, Lin XJ, Zhao JL, Shi W, Zhang H, Wang YS, Kan B, Du LC, Wang BD, Wei YQ, Liu Y and Zhao X: A tumorselective biotherapy with prolonged impact on established metastases based on cytokine gene-engineered MSCs. Mol Ther 16: 749-756, 2008.

20. Isaka N, Padera TP, Hagendoorn J, Fukumura D and Jain RK: Peritumor lymphatics induced by vascular endothelial growth factor-C exhibit abnormal function. Cancer Res 64: 4400-4404, 2004.

21. Tsopelas C, Bevington E, Kollias J, Shibli S, Farshid G, Coventry B and Chatterton BE: 99mTc-Evans blue dye for mapping contiguous lymph node sequences and discriminating the sentinel lymph node in an ovine model. Ann Surg Oncol 13: 692-700, 2006.

22. Partridge E, Kreimer AR, Greenlee RT, Williams C, Xu JL, Church TR, Kessel B, Johnson CC, Weissfeld JL, Isaacs C, Andriole GL, Ogden S, Ragard LR and Buys SS: Results from four rounds of ovarian cancer screening in a randomized trial. Obstet Gynecol 113: 775-782, 2009.

23. Sleeman JP: The lymph node as a bridgehead in the metastatic dissemination of tumors. Recent Results Cancer Res 157: 55-81, 2000.

24. Rissanen TT, Markkanen JE, Gruchala M, Heikura T, Puranen A, Kettunen MI, Kholova I, Kauppinen RA, Achen MG, Stacker SA, Alitalo K and Yla-Herttuala S: VEGF-D is the strongest angiogenic and lymphangiogenic effector among VEGFs delivered into skeletal muscle via adenoviruses. Circ Res 92: 1098-1106, 2003.

25. Skobe M, Hawighorst T, Jackson DG, Prevo R, Janes L, Velasco P, Riccardi L, Alitalo K, Claffey K and Detmar M: Induction of tumor lymphangiogenesis by VEGF-C promotes breast cancer metastasis. Nat Med 7: 192-198, 2001

26. Karpanen T, Egeblad M, Karkkainen MJ, Kubo H, YläHerttuala S, Jäättelä $M$ and Alitalo $\mathrm{K}$ : Vascular endothelial growth factor $\mathrm{C}$ promotes tumor lymphangiogenesis and intralymphatic tumor growth. Cancer Res 61: 1786-1790, 2001.

27. Shayan R, Achen MG and Stacker SA: Lymphatic vessels in cancer metastasis: bridging the gaps. Carcinogenesis 27 : 1729-1738, 2006. 
28. Ozols RF, Bookman MA, Connolly DC, Daly MB, Godwin AK, Schilder RJ, Xu X and Hamilton TC: Focus on epithelial ovarian cancer. Cancer Cell 5: 19-24, 2004.

29. Burke TG and Mi Z: The structural basis of camptothecin interactions with human serum albumin: impact on drug stability. J Med Chem 37: 40-46, 1994.

30. Wang XH, Cui FZ, Feng QL, Li JC and Zhang YH: Preparation and characterization of collagen/chitosan matrices as potential biomaterials. J Bioact Compat Polym 18: 453-467, 2003

31. Majeti NV and Ravi Kumar: A review of chitin and chitosan applications. React Funct Polym 46: 1-27, 2000.

32. Thanou M, Verhoef JC, Marbach P and Junginger HE: Intestinal absorption of octreotide: N-Trimethyl Chitosan Chloride (TMC) ameliorates the permeability and absorption properties of the somatostatin analogue in vitro and in vivo. J Pharm Sci 89: 951-957, 2000.
33. O'Grady A, Dunne C, O'Kelly P, Murphy GM, Leader M and Kay E: Differential expression of matrix metalloproteinase (MMP)-2, MMP-9 and tissue inhibitor of metalloproteinase (TIMP)-1 and TIMP-2 in non-melanoma skin cancer: implications for tumour progression. Histopathology 51: 793804,2007

34. Wu ZS, Wu Q, Yang JH, Wang HQ, Ding XD, Yang F and Xu XC: Prognostic significance of MMP-9 and TIMP-1 serum and tissue expression in breast cancer. Int $\mathrm{J}$ Cancer 122: 2050-2056, 2008 . 